\title{
Exploring personality-targeted UI design in online social participation systems
}

\author{
Oded Nov \\ Polytechnic Institute of \\ New York University \\ onov@poly.edu
}

\author{
Ofer Arazy \\ University of Alberta \\ ofer.arazy@ualberta.ca
}

\author{
Claudia López \\ University of \\ Pittsburgh \\ cal95@pitt.edu
}

\author{
Peter Brusilovsky \\ University of \\ Pittsburgh \\ peterb@pitt.edu
}

\begin{abstract}
We present a theoretical foundation and empirical findings demonstrating the effectiveness of personality-targeted design. Much like a medical treatment applied to a person based on his specific genetic profile, we argue that theorydriven, personality-targeted UI design can be more effective than design applied to the entire population. The empirical exploration focused on two settings, two populations and two personality traits: Study 1 shows that users' extroversion level moderates the relationship between the UI cue of audience size and users' contribution. Study 2 demonstrates that the effectiveness of social anchors in encouraging online contributions depends on users' level of emotional stability. Taken together, the findings demonstrate the potential and robustness of the interactionist approach to UI design. The findings contribute to the HCI community, and in particular to designers of social systems, by providing guidelines to targeted design that can increase online participation.
\end{abstract}

\section{Author Keywords}

Theory-driven design; user interface; personality; extroversion; emotional stability; audience size; anchoring.

\section{ACM Classification Keywords}

H.5.m. Information interfaces and presentation (e.g., HCI): Miscellaneous.

\section{General Terms}

Human Factors

\section{INTRODUCTION}

Understanding how psychology can inform the design of social technologies has been the subject of extant HCI research in recent years $[13,22,44,47]$. While the design of web-based applications to support social participation has been largely based on intuition, trial and error [43, 62], researchers of social participation in online settings have been trying to develop theories that will guide practice. To date, much of the HCI research in the area has focused on the general user population, overlooking personality

Permission to make digital or hard copies of all or part of this work for personal or classroom use is granted without fee provided that copies are not made or distributed for profit or commercial advantage and that copies bear this notice and the full citation on the first page. To copy otherwise, or republish, to post on servers or to redistribute to lists, requires prior specific permission and/or a fee.

CHI'13, April 27 - May 2, 2013, Paris, France.

Copyright 2013 ACM 978-1-XXXX-XXXX-X/XX/XX...\$10.00. differences. In these design-based studies, controlled experiments are used to test the effect of UI design features on user behavior, building on psychology theories to inform design choices $[13,22,47]$. This approach enables researchers to draw conclusions about design effectiveness. However, to date, studies based on this approach did not take into account differences in users' fundamental idiosyncratic attributes, such as their personalities or motivations; in other words, to follow a medical metaphor, this is equivalent to testing the effectiveness of a medical treatment by providing it to all patients, regardless of their specific genetic make-up.

We argue that an understanding of personality differences is essential for designing $\mathrm{HCI}$ for online participation, as different personalities are expected to respond differently to design cues. Drawing on the interactionist tradition in psychology research [17, 67], whereby situation and personality interact to determine people's behavior, we combine controlled experiments, design manipulations, surveys, and system data, to test the effectiveness of design features that target participants' idiosyncratic personal attributes. The Psychology literature is divided on the extent to which personality traits are viewed as stable and could predict behavior. While the "personality" approach focuses on individual differences as the primary predictor of behavior, the "situation" approach emphasizes on the characteristics of the situation where behavior takes place. The interactionist approach tries to bridge these opposing views and highlights the joint contribution of personality and situation factors to explaining human behavior $[17,35]$. As such, this perspective incorporates a broader range of explanations and allows researchers to test the effect of one set of variables while controlling for the other. The interactionist perspective has been applied in a variety of contexts to explain behaviors such as individuals' creativity [70] and reactions to organizational change [59].

\section{RELATED WORK}

Our approach to applying the interactionist perspective to the problem of online participation is inspired by the literature on individual differences, technology use and online engagement. Studies in that field showed how participants' personal attributes, such as motivations or personality traits, are correlated with technology use [49, $51,55]$ and online contribution $[25,57]$. 
Relevant to our research is the literature on personalization (e.g., [10, 23, 48]). Work in that area often develops a user model based on users' task-specific interactions (e.g. movie views by the user in a movie recommender system). In the present paper, on the other hand, we classify users based on pre-existing categories informed by psychology research (personality traits, in this case). In other words, while personalization often involves defining user personas based on task-specific prior activities, we define user profiles based on more fundamental user attributes that transcend specific settings.

In recent years, there is an attempt to explore personality differences within the context of HCI. For example, several studies have explored the interaction between users' personality and persuasive messages they receive. For example, [41] investigated the interaction between the trait of "persuadability" and health-related persuasive messages on people's participation in health-related activity; and [40] studied how the interaction between personality and influence strategies affects online purchase intentions. [34] took these ideas to the design of health-promoting mobile applications, and studied the effects of the interaction between personality and persuasive strategies on people's usage intentions. While such studies had the ultimate goal of informing design, they did not directly test the effects of UI design manipulations.

In the related field of adaptive UI, there has been prior experimental work on the interaction between personality traits and UI design features $[30,53]$. The primary objective of these studies has been to reduce users' cognitive load and make their interaction with the computer more efficient. The differences in goals between such studies and ours (reduce cognitive load vs. influence online participation), make them different in terms of the applicable design manipulations.

Building on these prior works, the goal of the present paper is to investigate the interaction between personality and design features on online participation.

\section{THE PROPOSED APPROACH}

Applying the interactionist approach to HCI design, we address the following general research question: can differences in users' idiosyncratic personal attributes explain the effects of design interventions on users' online participation? Using the medical metaphor mentioned above, the proposed approach is analogous to testing the effectiveness of a medical treatment being applied to individuals based on their specific genetic profile, rather than the same treatment applied to the entire population. While the proposed approach is applicable in a variety of design settings, we use as the setting of this research two social recommender systems, following the approach of MovieLens [25, 47], a social movie recommender system used as an online, live laboratory setting for researchers to test the effect of design on user behavior.
In a recent study [56], we reported a preliminary exploration of the interactionist approach, following the experimental design outlined in Table 1, in which we showed how users' conscientiousness levels determine their response to a particular design intervention (manipulating an indicator presenting the number of past contributors in an online setting). In the present study we explore new design contexts. We describe two studies in which we examine how the interaction between personality traits (extroversion in Study 1 and emotional stability in Study 2) and design interventions (experimental manipulation of perceived audience size and social anchoring in studies 1 and 2 respectively) affect user participation.

\begin{tabular}{|l|l|l|}
\cline { 2 - 3 } \multicolumn{1}{c|}{} & \multicolumn{2}{c|}{ Experimental manipulations } \\
\hline $\begin{array}{l}\text { Personal } \\
\text { Attributes }\end{array}$ & $\begin{array}{l}\text { Design } \\
\text { intervention: low } \\
\text { level }\end{array}$ & $\begin{array}{l}\text { Design } \\
\text { intervention: high } \\
\text { level }\end{array}$ \\
\hline $\begin{array}{l}\text { Low level } \\
\text { of personal } \\
\text { attribute }\end{array}$ & $\begin{array}{l}\text { Outcome for: } \\
\text { low attribute level } \\
\text { X low intervention }\end{array}$ & $\begin{array}{l}\text { Outcome for: } \\
\text { low attribute level } \\
\text { X high intervention }\end{array}$ \\
\hline $\begin{array}{l}\text { High level } \\
\text { of personal } \\
\text { attribute }\end{array}$ & $\begin{array}{l}\text { Outcome for: } \\
\text { high attribute level } \\
\text { X low intervention }\end{array}$ & $\begin{array}{l}\text { Outcome for: } \\
\text { high attribute level } \\
\text { X high intervention }\end{array}$ \\
\cline { 2 - 3 } & \multicolumn{2}{|c|}{ Experimental Outcomes } \\
\cline { 2 - 3 }
\end{tabular}

Table 1. Experimental design: attributes $X$ interventions.

This paper makes several contributions to HCI research. Study 1 helps explain the inconclusive evidence concerning users' response to UI indicator of group (or "audience") size, and shows that users' extroversion/introversion moderates the relationship between the UI manipulation and users' online participation. Study 2 expands the limited $\mathrm{HCI}$ literature on social anchoring. It demonstrates the effectiveness of UI indicators that serve as anchors in encouraging online contributions depends on users' level of emotional stability. Taking the results of these two studies together, we demonstrate the potential and robustness of the interactionist approach to UI design in different settings. We show that for different combinations of personality traits and design manipulation, users' personal idiosyncratic attributes play a significant role in determining the extent to which UI design manipulation encourage contribution in online social systems. Finally, our study contributes to $\mathrm{HCI}$ practitioners, and in particular to designers of recommender systems, by providing guidelines to targeted design that can increase online contributions.

In the sections that follow, we present two studies - the first focuses on the moderating effect of extroversion on the 
relationship between perceived audience size and online participation, and the second investigates the differential effects of social anchoring on online helping behavior, moderated by emotional stability. In each study we present the relevant literature, develop hypotheses about the factors affecting user behavior, test the hypotheses in an experimental setting, and discuss the findings briefly. We then conclude the paper with a broader discussion of the results, the interactionist approach to UI design, and the implications for theory and practice.

\section{STUDY 1: EXTROVERSION, AUDIENCE SIZE AND ONLINE CONTRIBUTION}

A large body of research on social technologies has been concerned with identifying effective ways to encourage contribution of public goods, such as users' knowledge in online settings [6, 7, 9]. A prominent avenue of research involved the analysis of the relationship between audience size and individuals' contribution levels [5, 7, 71]. However, research in this area offer inconsistent perspectives: for example, a study of Facebook newcomers [7] found a positive relation between perceived audience size and contribution levels, and [71] found that a reduction in the Chinese Wikipedia audience size (as a result of the blocking of Wikipedia in mainland China) led to a decrease in contributions among those users who were not blocked, suggesting a positive relation between contribution and audience size. Such empirical findings, however, stand in contrast with extant research on private provision of public goods, which suggests that the incentive to contribute is inversely related to group size due to free riding [71]. In many social technologies and online contribution settings (e.g., Wikipedia, yelp, open source software) a potential audience size is similar to the group size, since all readers can contribute and vice versa.

We offer a new perspective on such conflicting evidence, which allows for examining the response to UI indicators of audience size by different types of users. A fundamental factor that distinguishes individuals from one another is personality - the dispositions and interpersonal strategies that explain people's behavior, and the unique and relatively stable patterns of behaviors, shown by individuals [72]. A personality trait highly relevant to understanding users' response to the potential audience for their contribution is extroversion. Extroversion represents sociability, cheerfulness, and optimism, such that extroverts seek out new opportunities and excitement [51] and are sensitive to positive or pleasure cues in their social environment [12]. Introverts, on the other hand, are reserved and distant toward other people [32].

Extroversion has been associated with different patterns of Internet use [58]. Introverts were found to be more likely to prefer online to offline communication [16] and to spend more time on chat rooms than extroverts [4]. Introverts also reported that they can express themselves better in an online context than offline [3]. Introverts' preference for online communication can be explained by the "online disinhibition effect" theory [58]. This theory [66] states that some aspects of online communication may lead to less restrained communication and greater self-disclosure online. Among these characteristics, the dissociative anonymity, invisibility and asynchronicity aspects of online communication can make it more appealing to introverts. Being anonymous, remaining invisible to others, being unable to see others' physical communicational cues, and having time to think about what will be posted can help introverts to feel more comfortable online. On the other hand, other studies have found that extroverts were more likely than introverts to use the Internet to maintain their relationships with friends and family, to meet new people online and to frequent chat rooms [42]. Extroverts were also more likely to use social networking sites for maintaining relationships, entertainment and information expression [45]. An argument that can explain such results is that social networking sites are built around the user's preexisting offline social network [58]. This can make the factors that lead to the online disinhibition effect (e.g. anonymity) less salient, thus favoring extroverts rather than introverts

In the present study we test the hypothesis that extroverts will respond to a UI design feature indicating audience size in a different way than introverts. In other words, we hypothesize that users' extroversion level will moderate the relationship between perceived audience size and the likelihood they will participate in an online social setting.

We expect that a small audience size will be associated with lower contribution among extroverts and higher contribution among introverts. The reasoning behind this hypothesis is as follows: extroverts tend to be sociable, assertive, talkative and active, whereas introverts are shy and reserved [31]. Extroverts also tend to seek rewards, with little attention to the potential of negative consequences of such pursuit, whereas introverts are more oriented toward avoiding punishment than toward gaining rewards [32]. A large audience size may represent the potential benefit of making the contributor look knowledgeable and helpful among a large user population (or online "audience"), but also increases the potential for evaluation apprehension [15], whereby the presence of others leads people to refrain from expressing their views in social settings (e.g., classroom, meetings), because they are uncertain about how their views will be received [26]. Introverts, who seek to minimize the risks associated with their behavior, are expected to exhibit greater evaluation apprehension when the perceived audience size is larger (which increases the likelihood that the views they express will be received negatively by someone in the audience), and consequently, will tend to contribute less. Extroverts, on the other hand, will tend to play up the potential benefits of reaching a larger audience who may appreciate their contribution, and will therefore be more likely to participate 
when a larger audience is present. Furthermore, extroverts who tend to be sociable and relish the company of others are likely to be encouraged by the prospect of interacting with a large audience, whereas shy and reserved introverts may be intimidated by this prospect. We acknowledge that an alternative hypothesis might be that anonymity and identifiability in online communication can change group members' behavior. The Social Identity model of Deindividuation Effects $[60,61]$ and the online disinhibition effect theory have been used to explain how anonymity and identifiability in online communication can affect the group members differently than in offline communication and encourage participation among users such as introverts - who might be more inhibited in offline settings. The specific setting of our study, however, makes the latter hypothesis less applicable, given that all users come from the same graduate school, and therefore the perception of complete anonymity may be hard to maintain.

\section{Study 1: Methodology}

The experimental setting for testing the hypothesis in the present study was CourseAgent [21], a community-based study planning system for graduate students in the School of Information Sciences (iSchool) at the University of Pittsburgh. CourseAgent allows students to plan their studies and evaluate courses that they have taken, reflecting workload and relevance to personal career goals. The system serves as a source of knowledge about the suitability of graduate courses given specific career goals. Currently, the system has over 100 active users.

During the experiment period, the CourseAgent landing page (see Figure 1) invited participants to answer a short questionnaire which included personality items. Participants were surveyed only once during this period, and the response was measured dichotomously as providing - or not - at least one course evaluation. Self-report surveys are commonly used in social science research to identify personal attributes, and have been used extensively in $\mathrm{HCI}$ and $\mathrm{CSCW}$ studies [51, 65]. The two extroversion trait questionnaire items used a 7-point Likert scale and were taken from the Ten Item Personality Instrument [31], a short version of the Big Five instrument which has been validated and tested numerous times in prior studies.

The experimental manipulation included changing the UI indicators for audience size. A highly noticeable banner on the top of every interface of CourseAgent showed the number of visitors during the prior month. These numbers were manipulated to include two conditions - of small and large audience - applied in random. For the small-audience condition, the value assigned to the number of visitors was between 7 and 10 (values in this range applied randomly). For the large-audience condition, the number of visitors was 100 (ranging between 90-110). Figure 1 shows a screenshot of the large audience size condition. In summary, users were assigned to one of the four experimental conditions described in Table 1.

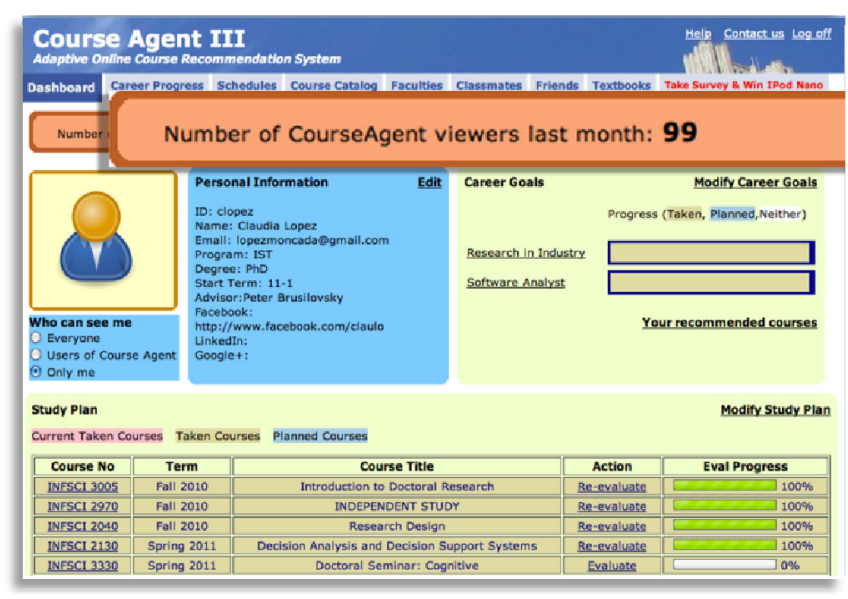

Figure 1. Audience size manipulation: large audience size.

Manipulation check: The low and high values of audience size (of roughly 10 and 100 respectively) were established after a pilot test conducted by using Amazon Mechanical Turk. In that experiment, participants were directed to a webpage describing a simple scenario. According to this scenario, the participant heard about a web site that provides movie recommendations based on users' ratings; when visiting the website, the participant finds out that its number of viewers last month was $X$ (where $X$ is manipulated by the researchers and is randomly assigned either the low or high audience size values). Having seen this, the participant in the pilot study is asked to what extent they agree with a statements that the movie reviews website has a large audience. Responses ranged between 1 (strongly disagree) to 7 (strongly agree) using a Likert scale. The mean responses of the populations' of the two experimental conditions were compared. 161 people took part in this pilot study. The low audience size indicator received the average score of 4.13 out of 7 , and the high audience size indicator received the average score of 4.94. A t-test was used to compare the means, and difference between them was found to be significant $(\mathrm{p}<0.001)$. The results supported the assumption that the two anchors represent large and small audience size.

\section{Study 1: Data analysis}

We first distinguished between extroverts and introverts by performing a median split, such that respondents whose extroversion score was above the sample median were classified as high-extroversion and those below the median as low-extroversion respondents. Cronbach's alpha of the extroversion responses was 0.77 , representing satisfactory reliability. The independent variables in the analysis included extroversion (high $=1$, low $=0$ ), perceived audience size (high $=1$, low $=0$ ), and the interaction between them. We were interested in understanding how the independent variables affect the likelihood that a user will decide to contribute. Therefore, the outcome variable was contribution, defined as providing at least one evaluation $($ Evaluated $=1$, Not evaluated $=0)$. In order to 
examine the effects of personality and design, as well as their interaction, and due to the dichotomous outcome variable, we used a factorial logistic regression in the statistical analysis.

\section{Study 1: Results and discussion}

Overall, 53 people participated in this study, of which 31 $(58.5 \%)$ were women. The average age was 28.3 (stdev=7.1). On average, participants contributed 1.9 evaluations and $43.4 \%$ of them contributed at least one evaluation. A between-subject comparison showed that contribution was characterized by power-law distribution. Examining the effect of the audience size alone, revealed no significant difference in contribution rate: $44.0 \%$ of those in the large audience condition and $42.9 \%$ of those in the large audience condition provided at least one evaluation. In other words, on average, audience size did not lead to more participation. However, as expected, a closer look at the participation rates of the sub-populations, considering both the external manipulation and the users' extroversion level, revealed a more intricate relationship (see Figure 2).

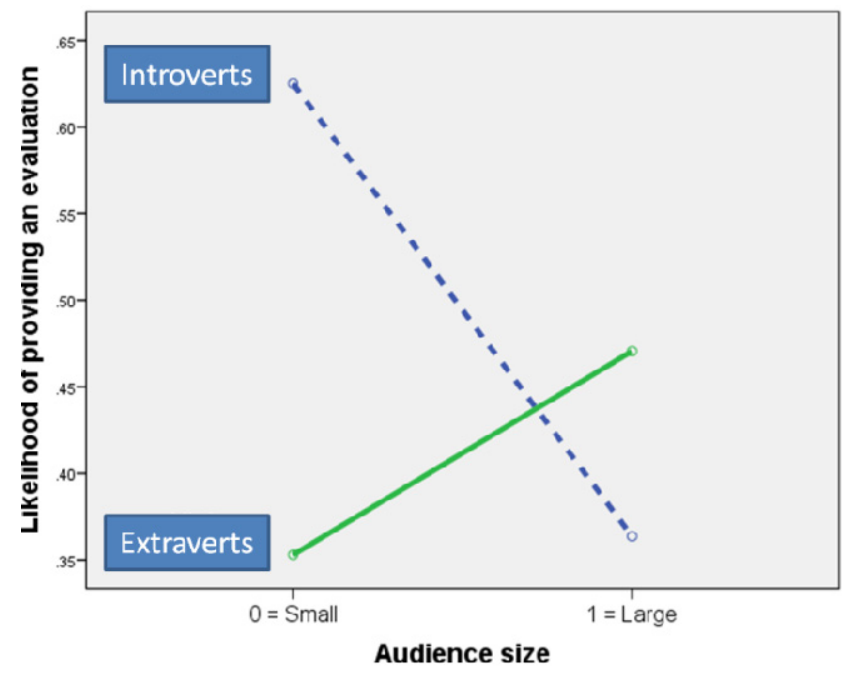

Figure 2. The differential effects of audience size: audience size and contribution are related positively among extroverts and negatively among introverts.

The results of the logistic regression showed that the main effects of the independent variables were not statistically significant $(\mathrm{B}=-.59, \mathrm{Wald}=.58, \mathrm{p}=.45$ for extroversion and $\mathrm{B}=-1.50$, Wald $=2.52, \mathrm{p}=.11$ for audience size). Examining the focus of our analysis, the interaction between audience size and extroversion level and its effect on contribution, was found to be significant, in the expected direction $(B=2.68$, Wald $=4.73, \mathrm{p}<.05)$.

The findings support the hypothesis that the interaction between extroversion and audience size is such that perceived large audience size is associated with increased contribution among extroverts and decreased contribution among introverts, whereas perceived low audience size will be associated with increased contribution by introverts and decreased contribution by introverts (see Figure 2).
The results call into question the effectiveness of a common practice in the design of social technologies, and highlight the potential effectiveness of a more nuanced personsituation interactionist approach: providing cues that emphasize the potential for a large audience size may be an effective way to increase participation among extroverts, but should be avoided when users are more introvert. Future work is needed in order to identify other personal attributes - such as social orientation or community commitment that may interact with perceived audience size. As a practical matter, surveying new users about their personality traits as part of their joining process could provide a minimally intrusive way to learn about users' personal attributes. With such information, social systems' designers can develop adaptive user experience, which include theory-driven design cues based the idiosyncratic personal attributes of users.

\section{STUDY 2: EMOTIONAL STABILITY, SOCIAL ANCHORING AND CONTRIBUTION}

Human judgment tends to be influenced by anchoring: when asked to make a quantitative judgment, people are often influenced by externally presented information when such information is available to them [52]. Anchoring is seen as one of three basic heuristics in intuitive judgment [39]. Extant psychology research demonstrates the cognitive effects of anchoring: experimental manipulation of the initial values, or anchors, leads to estimates that are biased toward the anchor $[18,28]$. As a result, studies of the effects of anchoring on human behavior were carried out in a variety of disciplines, including finance [36], law [33] and marketing [1]. Some of the psychological mechanisms underlying anchoring are confirmatory hypothesis testing, numeric or magnitude priming, and insufficient adjustment. Recent studies took a broader view of anchoring and adopted an attitudes and persuasion perspective, focusing on the social context in which anchors arise [69]. We use the term social anchoring to refer to an anchoring effect where the social context, and in particular the anchor's source, elicit processes of persuasion and social influence and affect judgment [19].

Generally speaking, HCI research on the effects on anchoring has been relatively scarce [2], with the notable exception of [11], who found that when users of a movie recommender system were asked to re-rate movies while (experimentally manipulated) "predicted" rating were presented to them, they tended to change their rating toward the "prediction" anchor. More recently, [2] showed that users' ratings can be influenced by a recommender system's (experimentally manipulated) anchors, and that the effects of anchoring can be separated from the effects of the system's perceived reliability. We build on this prior work and extend it to explore whether some people are more sensitive to anchoring than others. 
The interaction between personality traits and anchoring has been the subject of recent research. For example, it was shown that individuals who are high in the openness-toexperience personality trait were significantly more influenced by anchoring cues relative to participants low in this trait [52], and [20] found that those high on agreeableness and conscientiousness but low on extroversion were more susceptible to anchoring. In contrast, [27] found no significant interaction between anchoring cues and the personality traits of openness-toexperience. Our focus is on the personality trait of emotional stability, sometimes known as the opposite of neuroticism $[54,68]$, which we take to be highly relevant for the anchoring context: people characterized by high emotional stability tend to be secure and self-assured [12], whereas those who are low in emotional stability (i.e. high in neuroticism) tend to be insecure and self-doubting and experience the world as distressing [14]. We argue that emotional stability has the potential to explain user behavior in the presence of anchoring: since individuals who are high in emotional stability tend to be more secure and self-assured, we expect that they will be less susceptible to the influence of social anchoring cues. Individuals who are high on neuroticism, on the other hand, tend to have an external control of reinforcement [37, 38]. Internal versus external control (i.e. 'locus of control') refers to the degree to which people believe events in their life and rewards they receive are the result of their own behavior or outside of their control [63, 64]. Hence, because those high in neuroticism tend to be externally focused, we expect them to be more susceptible to the influence of others, and in particular to be more influenced by anchors representing the opinions of others. In sum, we hypothesize that the effect of social anchoring cues on users' rating will be weaker among high- emotional stability participants compared to low- emotional stability participants.

\section{Study 2: Methodology}

We tested our hypothesis using a simulated online recommender system called PetLink which we developed as an experimental platform. PetLink is presented as a research project in need of feedback from users: its landing page invites participants to answer a very short personality questionnaire, and a simulated recommender system returns a picture of a pet that purports to present the "best match" for the participant's personality (see Figures 3-5). The PetLink environment facilitates the implementation of a 2X2 factorial experimental design illustrated in Table 1, consisting of: (1) measurement of personal attributes (through a short questionnaire), (2) design intervention, and (3) opportunities for users to rate the accuracy of the recommendation and provide feedback. As such, PetLink serves as an experimental platform through which we can address research questions on the interactions between personal attributes, design choices, and user behavior.

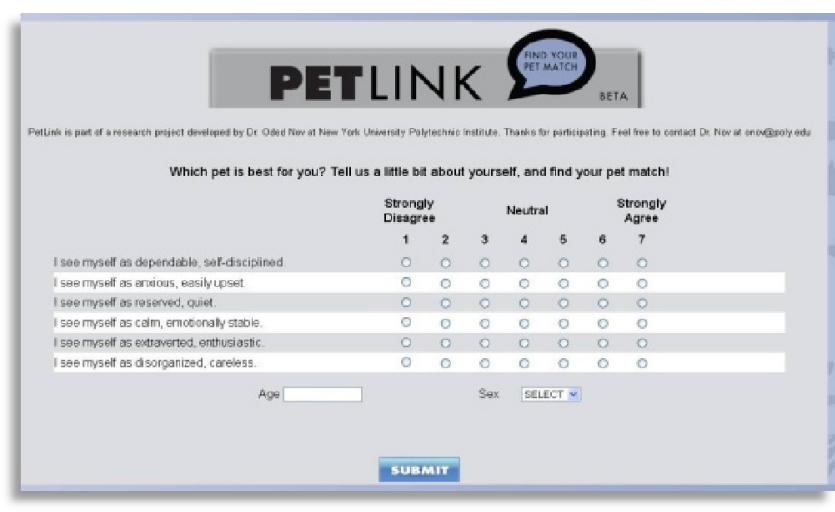

Figure 3. PetLink landing page screenshot.

Participants in the study were recruited via Amazon Mechanical Turk. They received $\$ 0.05$ and took part in the study only once. Participants were led to believe that PetLink is part of an academic research project involving the development of a technique to match respondents' personality traits and the pet that would suit them most. This was communicated to participants in a recruitment announcement, and on the PetLink site itself.

The PetLink landing page (see Figure 3) invited participants to answer a short personality questionnaire. The two emotional stability questionnaire items used a 7-point Likert scale and were adapted from the Ten Item Personality Instrument [31], a short version of the Big Five instrument.

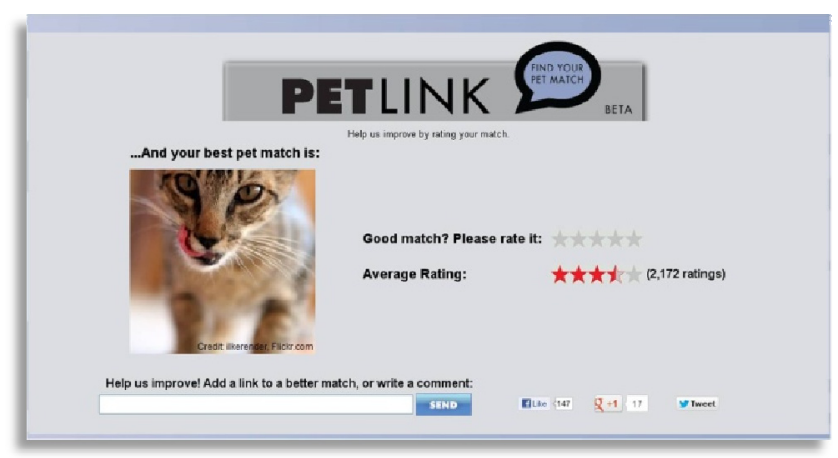

Figure 4. PetLink image page screenshot.

After filling out the short questionnaire respondents were presented with their purported "best match" (see Figure 4): an image of an animal based on the responses to the survey questions, such that each combination of responses was associated with a specific pet image. Unbeknown to the respondents, the system consistently, but arbitrarily, paired certain images with certain personality profiles, with no attempt to match images to personalities. At this stage, respondents were requested to rate the quality of the match on a five-star scale (see Figure 4). In line with the experimental design presented in Table 1, a median split was performed such that respondents with an emotional stability score above the sample median were classified as 
high- emotional stability participants, and those below the median as low- emotional stability.

The experimental manipulation consisted of high and low social anchor level: In addition to the image, respondents were also presented with information about the average rating for the particular pet image presented to them (along the lines of UI design common on popular recommender systems such as Amazon or Netflix). The "Average Rating" value (see Figure 5), representing the social anchor, was experimentally manipulated and randomly assigned either high level (4.5 stars) or low level (1 star).

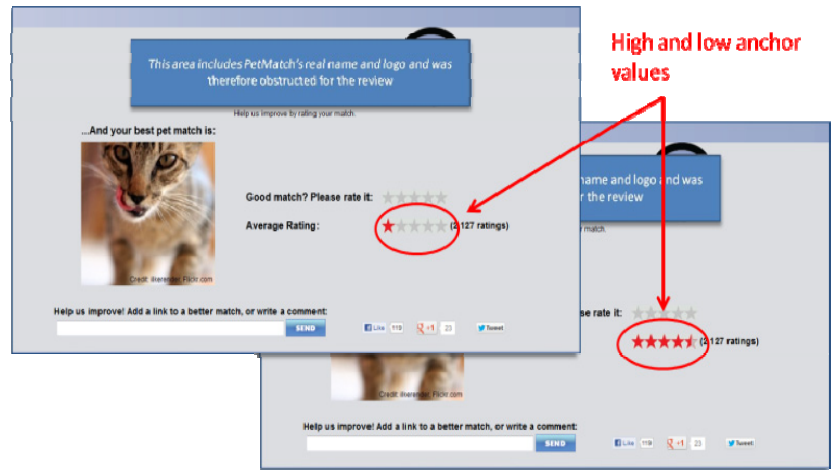

Figure 5. Experimental manipulation: two anchor values.

In summary, at this stage all users were assigned to one of the four experimental conditions described in Table 1, and following a between-subjects study design, their responses were compared.

\section{Study 2: Data analysis}

Overall, 375 people participated in this study, of which $47.2 \%$ were women. The average age was 28.7 ( $\mathrm{stdev}=9.9)$. 249 participants $(66.4 \%)$ provided rating. Cronbach's alpha of the emotional stability responses was 0.74 , representing satisfactory reliability. To test our hypothesis that anchoring is less effective among people with high emotional stability, we ran a regression with the independent variables of emotional stability (high emotional stability $=1$, low emotional stability $=0$ ), perceived social anchor (high $=1$, low $=0$ ), and the interaction between them. The outcome variable was the average rating (ranging between 1-5 stars) provided by the participants.

\section{Study 2: Results and discussion}

The average rating among participants in all experimental conditions was 3.04. Consistent with prior studies, ratings were biased toward the anchors, with mean rating $=3.36$ among high-anchor participants and 2.71 and among low anchor participants. The results of the regression revealed an insignificant main effect of emotional stability $(p=.6)$ and a significant effect of perceived social anchor $(\mathrm{p}<.01)$. As expected, both the regression and an ANOVA revealed that the interaction between the independent variables was significant $(\mathrm{p}<.05)$, thus supporting our hypothesis: on average, all participants were susceptible to anchoring (i.e., provided higher rating when shown the high social anchor), however, the effect of anchoring on high emotional stability participants was weaker than its effect on low emotional stability (neurotic) participants (see Figure 6).

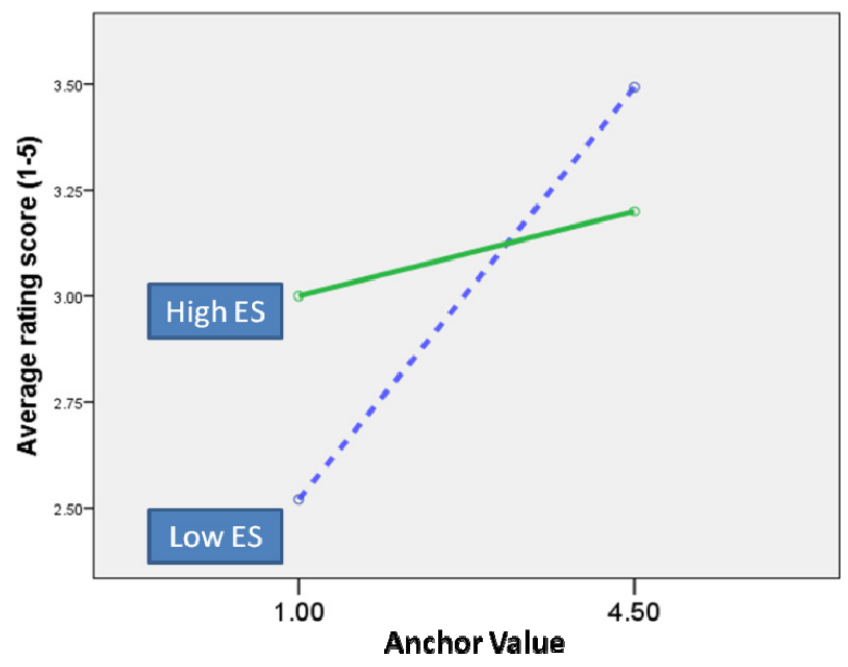

Figure 6. The differential effect of social anchoring: high Emotional Stability participants are less susceptible to social anchoring than low Emotional Stability participants.

The results highlight the potential effectiveness of the personality targeted interactionist approach: anchoring may be a universal phenomenon, but its magnitude is highly person-specific. From an interaction design perspective, using anchors as a way to influence behavior is more effective among some users, and further work is needed to identify other personal attributes that may influence the effect of anchoring. Another related open question concerns the identification of other UI design interventions that may interact with the Emotional Stability personality trait.

\section{OVERALL DISCUSSION AND CONCLUSIONS}

In the two studies described here, we tested the effectiveness of personality-targeted design approach as a way to influence participants and increase their participation. Specifically, by focusing on audience size and social anchoring cues, we sought to examine relatively popular HCI design practices (namely, displaying others' behavior, including others' rating and the number of others' prior visits), in order to (1) demonstrate how insights from psychology research can be used in novel ways in an $\mathrm{HCI}$ context to improve the effectiveness of social technologies, and (2) use HCI design as a large-scale experimental tool through which to contribute to psychology research.

The UI design cues we tested were shown to have a differential effect on participants with different levels of the extroversion and emotional stability, supporting our hypotheses and illustrating the potential value of personality-targeted design.

While specific design implications were discussed for each study separately, a more general implication from the findings is that we cannot necessarily expect indicators of 
community's activity (such as audience size, or others' rating) to equally affect all participants . Instead, a more nuanced, targeted approach to UI design is needed. This is made particularly evident in the case of Study 1, where displaying the number of other users' past visits led to effects in opposite directions (see Figure 2). These effects cancel each other out when not considering the personal attributes of users. In other words, certain design intervention may seem ineffective, whereas a closer look at the interaction between these intervention and the users' attributes will reveal effective ways to encourage contribution.

More broadly, our proposed approach to targeted design highlights the need to tailor design features to personality or other idiosyncratic personal characteristics, such that frontend and back-end design features will cater to users' particular personal attributes. In recent years, a number of studies have investigated the effects of users' personal traits on UI design. Studies on persuasion strategies have shown how personality determines people's reaction to persuasive messages [40] and have suggested that this approach is applicable to the design of system interfaces [34]. Studies on adaptive UI have demonstrated that personality-based design can reduce users' cognitive load [30, 53]. Our studies build on and extend such prior work, showing that personality-targeted design can enhance online participation.

Another implication of our results is the need to explore systems that can automatically adapt their features to users' personal attributes. In the area of e-learning, where surveybased assessment of learner individual traits is common, research on adaptation to individual traits has already become popular [24]. To reduce users' burden of answering long personality questionnaire, designers may survey new users as part of their joining the system, or make it part of a game-like activity (such as PetLink).

Recent research opens interesting prospects for unobtrusive adaptation to user personal traits. Since the survey approach used in the present study may be less appropriate in other kinds of interactive systems, there are less intrusive methods for automatically detecting aspects of users' personality. Such methods may include a measurement of invariable traits and dispositions (e.g., by creating a user profile), or even capturing users' transient preferences and attitudes such that the UI is not only personalized across users but also tailored to a users' particular attitude at particular points in time. Recent research has demonstrated the feasibility of recognizing user traits in "rich" multimodal and dialog interfaces $[30,46,50]$, and in Web and mobile phone interfaces [8, 29].

A limitation of the studies presented here stems from their experimental design: by exploring one design intervention and one personal attribute at a time, we limit the scope of the findings. Future work may address this by examining simultaneously a larger number of interventions and personal attributes. Another limitation is the measurement of personal attributes at a single point in time.

Future research may also involve the differential effects of a larger set of design interventions in a wider range of contexts, including mobile and haptic interfaces. It may also consider a wider range of personal attributes, beyond personality traits, such as motivations and context-specific attitudes. The present study demonstrates the utility of considering such factors, simultaneously, in the design of social technologies.

\section{ACKNOWLEDGMENTS}

This work was partially supported by NSF Award IIS 1149745, by the Fulbright Program and by CONICYT, Chile. We thank Erez Heiman for site design, and Durga Bhavani and Manasa Ranjitha Alla for research assistance.

\section{REFERENCES}

[1] Adaval, R. and Wyer Jr, R. S., "Conscious and Nonconscious Comparisons with Price Anchors," Journal of Marketing Research, 48, 355-365, 2011.

[2] Adomavicius, G., Bockstedt, J., Curley, S., and Zhang, J., "Recommender systems, consumer preferences, and anchoring effects," Proceedings of the RecSys Workshop on Human Decision Making in Recommender Systems, 2011.

[3] Amichai-Hamburger, Y., Wainapel, G., and Fox, S., ""On the Internet no one knows I'm an introvert"," CyberPsychology \& Behavior, 5, 125-128, 2002.

[4] Anolli, L., Villani, D., and Riva, G., "Personality of people using Chat," CyberPsychology \& Behavior, 8, 8995, 2005.

[5] Antin, J. and Cheshire, C., "Readers are not free-riders: reading as a form of participation on wikipedia," Proceedings of the ACM conference on Computer Supported Cooperative Work, Savannah, GA, 2010.

[6] Antin, J., Cheshire, C., and Nov, O., "Technologymediated contributions: editing behaviors among new wikipedians," Proceedings of the ACM conference on Computer Supported Cooperative Work (CSCW), Seatttle, WA, 2012.

[7] Burke, M., Marlow, C., and Lento, T., "Feed me: motivating newcomer contribution in social network sites," Proceedings of the 27th international Conference on Human Factors in Computing Systems 2009.

[8] Chittaranjan, G., Blom, J., and Gatica-Perez, D., "Mining large-scale smartphone data for personality studies," Personal and Ubiquitous Computing, 1-18, 2012.

[9] Choi, B., Alexander, K., Kraut, R. E., and Levine, J. M., "Socialization tactics in wikipedia and their effects," Proceedings of the 2010 ACM conference on Computer Supported Cooperative Work (CSCW), 2010.

[10] Chu, W. and Park, S.-T., "Personalized recommendation on dynamic content using predictive bilinear models," Proceedings of the 18th International Conference on the World Wide Web (WWW), Madrid, Spain, 2009. 
[11] Cosley, D., Lam, S. K., Albert, I., Konstan, J. A., and Riedl, J., "Is seeing believing?: how recommender system interfaces affect users' opinions," Proceedings of the SIGCHI conference on Human factors in computing systems, Ft. Lauderdale, FL, 2003.

[12] Costa, P. and McCrae, R., "NEO Personality Inventory-Revised (NEO-PI-R) and NEO Five-Factor Inventory (NEO-FFI) Professional Manual," Odessa, FL: Psychological Assessment Resources, 1992.

[13] Dabbish, L. and Kraut, R., "Awareness displays and social motivation for coordinating communication," Information Systems Research, 19, 221-238, 2008.

[14] Diefendorff, J. M. and Richard, E. M., "Antecedents and consequences of emotional display rule perceptions," Journal of Applied Psychology, 88, 284, 2003.

[15] Diehl, M. and Stroebe, W., "Productivity loss in brainstorming groups," Journal of Personality and Social Psychology, 53, 497-509, 1987.

[16] Ebeling-Witte, S., Frank, M., and Lester, D., "Shyness, Internet use, and personality," CyberPsychology \& Behavior, 10, 713-716, 2007.

[17] Endler, N. and Parker, J., "Interactionism revisited," European Journal of Personality, 6, 177-198, 1992.

[18] Englich, B., Mussweiler, T., and Strack, F., "Playing dice with criminal sentences," Personality and Social Psychology Bulletin, 32, 188-200, 2006.

[19] Epley, N. and Gilovich, T., "Anchoring unbound," Journal of Consumer Psychology, 20, 20-24, 2010.

[20] Eroglu, C. and Croxton, K. L., "Biases in judgmental adjustments of statistical forecasts," International Journal of Forecasting, 26, 116-133, 2010.

[21] Farzan, R. and Brusilovsky, P., "Encouraging user participation in a course recommender system: An impact on user behavior," Computers in Human Behavior, 27, 276-284, 2011.

[22] Farzan, R., Dabbish, L., Kraut, R., and Postmes, T., "Increasing commitment to online communities by designing for social presence," ACM 2011 Conference on Computer Supported Cooperative Work (CSCW), Hangzhou, China, 2011.

[23] Felfernig, A., Mandl, M., Tiihonen, J., Schubert, M., and Leitner, G., "Personalized user interfaces for product configuration," Proceedings of the 15th International Conference on Intelligent User Interfaces, Hong Kong, China, 2010.

[24] Ford, N. and Chen, S., "Individual differences, hypermedia navigation, and learning," Journal of educational multimedia and hypermedia, 9, 281-311, 2000.

[25] Fugelstad, P., Dwyer, P., Filson Moses, J., Kim, J., Mannino, C. A., Terveen, L., and Snyder, M., "What makes users rate (share, tag, edit...)?: predicting patterns of participation in online communities," Proceedings of the ACM 2012 conference on Computer Supported Cooperative Work, 2012.
[26] Furnham, A. and Yazdanpanahi, T., "Personality differences and group versus individual brainstorming," Personality and Individual Differences, 19, 73-80, 1995.

[27] Furnham, A., Boo, H. C., and McClelland, A., "Individual differences and the susceptibility to the influence of anchoring cues," Journal of Individual Differences, 33, 89, 2012.

[28] Galinsky, A. D. and Mussweiler, T., "First offers as anchors," Journal of Personality and Social psychology, 81, 657, 2001.

[29] Golbeck, J., Robles, C., Edmondson, M., and Turner, K., "Predicting personality from twitter," IEEE International Conference on Privacy, security, risk and trust (PASSAT), 2011.

[30] Goren-Bar, D., Graziola, I., Pianesi, F., and Zancanaro, M., "The influence of personality factors on visitor attitudes towards adaptivity dimensions for mobile museum guides," User Modeling and User-Adapted Interaction, 16, 31-62, 2006.

[31] Gosling, S., Rentfrow, P., and Swann, W., "A very brief measure of the Big-Five personality domains," Journal of Research in Personality, 37, 504-528, 2003.

[32] Graziano, W. G., Feldesman, A. B., and Rahe, D. F., "Extroversion, social cognition, and the salience of aversiveness in social encounters," Journal of Personality and Social psychology, 49, 971, 1985.

[33] Guthrie, C., Rachlinski, J., and Wistrich, A., "Blinking on the bench," Cornell Law Review, 93, 1, 2007.

[34] Halko, S. and Kientz, J., "Personality and persuasive technology: An exploratory study on health-promoting mobile applications," Persuasive technology, 6137, 150$161,2010$.

[35] Hough, L. M. and Furnham, A., "Use of personality variables in work settings," Handbook of psychology, 2003.

[36] Johnson, J. E. V., Schnytzer, A., and Liu, S., "To what extent do investors in a financial market anchor their judgments excessively?," Journal of Behavioral Decision Making, 22, 410-434, 2009.

[37] Judge, T., "Core self-evaluations and work success," Current Directions in Psychological Science, 18, 58-62, 2009.

[38] Judge, T., Erez, A., Bono, J., and Thoresen, C., "Are measures of self-esteem, neuroticism, locus of control, and generalized self-efficacy indicators of a common core construct?," Journal of personality and social psychology, 83, 693-710, 2002.

[39] Kahneman, D., Slovic, P., and Tversky, A., Judgment under uncertainty: Heuristics and biases: Cambridge University Press, 1982.

[40] Kaptein, M. and Eckles, D., "Heterogeneity in the effects of online persuasion," Journal of Interactive Marketing, 26, 176-188, 2012.

[41] Kaptein, M., Lacroix, J., and Saini, P., "Individual differences in persuadability in the health promotion domain," Persuasive technology, 6137, 94-105, 2010. 
[42] Kraut, R., Kiesler, S., Boneva, B., Cummings, J., Helgeson, V., and Crawford, A., "Internet Paradox Revisited," Journal of Social Issues, 58, 49-74, 2002.

[43] Kraut, R., Maher, M. L., Olson, J., Malone, T. W., Pirolli, P., and Thomas, J. C., "Scientific Foundations: A Case for Technology-Mediated Social-Participation Theory," IEEE Computer, 43, 22-28, 2010.

[44] Kraut, R. E. and Resnick, P., Evidence-based social design: MIT Press, 2011.

[45] Langstedt, E., "An Examination of the Five Factor Model Personality Traits as Predictors of Online Social Network Use," University of Connecticut, 2011.

[46] Lepri, B., Mana, N., Cappelletti, A., Pianesi, F., and Zancanaro, M., "Modeling the personality of participants during group interactions," User Modeling, Adaptation, and Personalization, 114-125, 2009.

[47] Ling, K., Beenen, G., Ludford, P., Wang, X., Chang, K., Li, X., Cosley, D., Frankowski, D., Terveen, L., and Rashid, A., "Using Social Psychology to Motivate Contributions to Online Communities," Journal of Computer-Mediated Communication, 10, 2005.

[48] Liu, J., Dolan, P., and Pedersen, E., "Personalized news recommendation based on click behavior," Proceedings of the 15th International Conference on Intelligent User Interfaces, Hong Kong, China, 2010.

[49] Ludford, P. and Terveen, L., "Does an individual's Myers-Briggs type indicator preference influence taskoriented technology use?," Interact2003. Proceedings of the Ninth IFIP TC13 International Conference on HumanComputer Interaction, 2003.

[50] Mairesse, F., Walker, M., Mehl, M., and Moore, R., "Using linguistic cues for the automatic recognition of personality in conversation and text," Journal of Artificial Intelligence Research, 30, 457-500, 2007.

[51] McElroy, J., Hendrickson, A., Townsend, A., and DeMarie, S., "Dispositional factors in internet use," MIS Quarterly, 31, 809-820, 2007.

[52] McElroy, T. and Dowd, K., "Susceptibility to anchoring effects," Judgment and Decision Making, 2, 4853, 2007.

[53] McGrenere, J., Baecker, R., and Booth, K., "An evaluation of a multiple interface design solution for bloated software," Proceedings of the SIGCHI conference on Human factors in computing systems, 2002.

[54] Mobbs, D., Hagan, C. C., Azim, E., Menon, V., and Reiss, A. L., "Personality predicts activity in reward and emotional regions associated with humor," Proceedings of the National Academy of Sciences, 102, 16502, 2005.

[55] Nov, O. and Ye, C., "Users' personality and perceived ease of use of digital libraries: The case for resistance to change," Journal of the American Society for Information Science and Technology, 59, 845-851, 2008.

[56] Nov, O. and Arazy, O., "Personality-Targeted Design: Theory, Experimental Procedure, and Preliminary Results," Proceedings of the ACM Conference on Computer Supported Cooperative Work (CSCW 2013) San Antonio, TX, 2013.
[57] Nov, O., Naaman, M., and Ye, C., "What drives content tagging: the case of photos on Flickr," Proceedings of the 26th annual SIGCHI Conference on Human Factors in Computing Systems 2008.

[58] Orchard, L. J. and Fullwood, C., "Current Perspectives on Personality and Internet Use," Soc. Sci. Comput. Rev., 28, 155-169, 2010.

[59] Oreg, S., "Personality, context, and resistance to organizational change," European Journal of Work and Organizational Psychology, 15, 73-101, 2006.

[60] Postmes, T., Spears, R., and Lea, M., "Breaching or Building Social Boundaries?: SIDE-Effects of ComputerMediated Communication," Communication Research, 25, 689-715, December 1, 19981998.

[61] Postmes, T., Spears, R., Sakhel, K., and de Groot, D., "Social Influence in Computer-Mediated Communication," Personality and Social Psychology Bulletin, 27, 12431254, 2001

[62] Preece, J. and Shneiderman, B., "The Reader-toLeader Framework," AIS Transactions on HumanComputer Interaction, 1, 13-32, 2009.

[63] Rotter, J. B., "Some problems and misconceptions related to the construct of internal versus external control of reinforcement," Journal of consulting and clinical psychology, 43, 56-67, 1975.

[64] Rotter, J. B., "Internal versus external control of reinforcement: A case history of a variable," American psychologist, 45, 489-493, 1990.

[65] Seay, A. and Kraut, R., "Project massive: selfregulation and problematic use of online gaming," Proceedings of the SIGCHI conference on human factors in computing systems, 2007.

[66] Suler, J., "The online disinhibition effect.," CyberPsychology \& Behavior, 7, 312-326, 2004.

[67] Swann, W. B. and Seyle, C., "Personality psychology's comeback and its emerging symbiosis with social psychology," Personality and Social Psychology Bulletin, 31, 155-165, 2005.

[68] Vittersu, J., "Personality traits and subjective wellbeing:: emotional stability, not extroversion, is probably the important predictor," Personality and Individual Differences, 31, 903-914, 2001.

[69] Wegener, D., Petty, R., Blankenship, K., and Detweiler-Bedell, B., "Elaboration and numerical anchoring," Journal of Consumer Psychology, 20, 5-16, 2010.

[70] Woodman, R. and Schoenfeldt, L., "An Interactionist Model of Creative Behavior," The Journal of Creative Behavior, 24, 279-290, 1990.

[71] Zhang, X. and Zhu, F., "Group size and incentives to contribute," The American economic review, 101, 1601$1615,2011$.

[72] Zweig, D. and Webster, J., "What are we measuring? An examination of the relationships between the big-five personality traits, goal orientation, and performance intentions," Personality and Individual Differences, 36, 1693-1708, 2004. 